\title{
INTESTINAL OBSTRUCTION AS A LATE COMPLICATION OF FIBROCYSTIC DISEASE OF THE PANCREAS (MUCOSIS)
}

\author{
BY \\ O. D. FISHER \\ From the Royal Belfast Hospital for Sick Children
}

(RECEIVED FOR PUBLICATION FEBRUARY 8, 1954)

The features of fibrocystic disease of the pancreas are well recognized following the description by Andersen in 1938 of three main clinical forms.

The first is that of intestinal obstruction in the first few days of life due to meconium ileus. About a fifth of all cases present with failure to pass meconium which is followed by vomiting and abdominal distension. It results from inspissation of meconium in the small bowel and at operation or necropsy the upper part of the small bowel is seen to be distended with gas and fluid. The lower part is filled with hard inspissated meconium and the bowel wall hypertrophies and tapers into an unexpanded ribbon-like colon. Perforation of the bowel wall may occur causing meconium peritonitis while volvulus and sometimes atresia of the small bowel may result. Bodian (1952) describes three instances of meconium retention in which spontaneous recovery occurred a few days after birth although all finally succumbed to the disease.

The second form is that of respiratory disorders which is marked by a distressingly persistent and spasmodic cough which simulates pertussis and is associated with episodes of respiratory infection usually due to Staphylococcus aureus. Respiratory distress with an increased respiratory rate, signs of infection and emphysema may be noted. Bronchiectatic changes are common and finger clubbing and even cor pulmonale may develop.

The third form is that of the coeliac syndrome in which failure to thrive in spite of an excellent appetite is followed by loose offensive stools, gaseous abdominal distension, wasting and stunting.

Meconium ileus is usually fatal though immediate survival has followed surgical intervention but later features of the disease soon supervene. Numerous variations and combinations of symptoms may confuse the clinical picture but, despite fluctuations, the course of the disease is invariably fatal.
In fibrocystic disease of the pancreas, intestinal obstruction after the neonatal period due to inspissated small bowel contents with recovery is considered worth while reporting.

\section{Case Report}

John C. was first admitted to hospital on December 8, 1951 , aged 13 months, with a persistent cough and failure to thrive. Birth weight was $6 \mathrm{lb}$. and after two months of breast feeding, dried milk and later raw cow's milk feeds were substituted. At 3 months a spasmodic but persistent cough resembling pertussis developed. At 6 months mixed feeding was started, and in spite of an excellent appetite the child did not thrive, having loose, offensive and frequent stools.

Of the family, the parents and three brothers are alive and well, two sisters and a brother died at 2 months, 6 months and 4 months; each child failed to thrive and developed loose, offensive stools and a cough like that of pertussis which led to a terminal respiratory illness.

On examination, the child was marasmic, weighing $12 \frac{1}{2} \mathrm{lb}$., with abdominal distension and a loose cough associated with scattered rhonchi in the chest. Investigation showed no tryptic activity in the stools or in three specimens of duodenal juice. The Mantoux reaction was negative. Staphylococcus aureus was cultured from the nasopharynx and a radiograph of the chest showed emphysema and heavy hilar shadows. Treatment with aureomycin and pancreatin resulted in marked improvement and a gain of $2 \mathrm{lb}$. in weight in 3 weeks. The child was discharged on January 4, 1952, on a high-protein diet with pancreatin granules, 45 gr., before each feed and aureomycin, $250 \mathrm{mg}$. daily.

Progress was satisfactory until February 4, 1952, when the supply of pancreatin ran out and could not be replenished. The next day the child vomited and later developed abdominal pain. On February 9, when readmitted to hospital aged 15 months, vomiting was continuous and, except for one small stool two days before, constipation was complete.

The child was irritable with bouts of abdominal pain causing the legs to be drawn up on to the abdomen. The latter was grossly distended but resonant. Bowel 
sounds but no visible peristalsis were present. An indefinite mass was present in the right iliac fossa, the rectum was empty and no blood or mucus was present or had been passed.

Acute intestinal obstruction was diagnosed and immediate laparotomy undertaken. On opening the peritoneal cavity a small amount of clear fluid was seen; the whole of the small bowel was found to be intermittently obstructed by masses of putty-like material in the bowel lumen. It was so firm that it could not be expressed through the ileocaecal valve. The large bowel was unobstructed. A portion of the small bowel was packed off and an incision made through the bowel wall; from the lumen a tenacious material like liquid rubber was extracted. The incision was repaired and as the whole of the small bowel was affected no further operative procedure was undertaken.

Post-operatively hydration was maintained by intravenous and subcutaneous fluids. After gastric aspiration $45 \mathrm{gr}$. of pancreatin granules freshly dissolved in a small quantity of water were given at four-hourly intervals. A daily bowel wash-out was given and on February 16 a large bowel action followed with relief of abdominal distension and vomiting.

From February 14, terramycin, $50 \mathrm{mg}$., was given orally twice a day. The child made an uninterrupted recovery and was discharged to continue on a high-protein diet with pancreatin $45 \mathrm{gr}$. before each feed. On July 21 , 1952, the child was again admitted with a further respiratory infection which responded to aureomycin therapy. Investigations again confirmed the lack of tryptic activity in stools and duodenal juice.

\section{Discussion}

This child's illness illustrates the customary course of fibrocystic disease of the pancreas with the early onset of respiratory and alimentary symptoms. The family history suggests that three siblings suffered from the same disease which is known to be inherited as a recessive characteristic. The diagnosis was confirmed by the lack of tryptic activity in the stools and duodenal juice on repeated examinations. The development of intestinal obstruction due to inspissated small bowel material has been reported only once before as a late complication in fibrocystic disease of the pancreas.

Levy (1951) records such an instance in a child at 7 months of age. Fibrocystic disease of the pancreas had been diagnosed at 9 weeks and treated with pancreatin, 60 gr. daily. When acute intestinal obstruction developed a tentative diagnosis of intussusception was made and laparotomy performed. The small bowel was found to be distended and plum-coloured, with haemorrhages in the root of the mesentery; the colon was empty and contracted. No surgical procedure was undertaken and the child died the following day. At necropsy addition to the laparotomy findings, the small bowel was filled with putty-like material. There was no evidence of volvulus, intussusception or any other organic obstruction present. The pancreas was firmer than normal and showed histologically the features characteristic of fibrocystic disease with a great increase in fibrous tissue, cystic dilatation of the ducts which contained desquamated epithelium and homogeneous eosinophilic tissue.

These two cases closely parallel those of meconium ileus with the inspissated gut contents causing intestinal obstruction which affects the small bowel leaving the large bowel collapsed but unobstructed. In meconium ileus the primary cause of obstruction is the abnormal physical character of the meconium, suggested by Landsteiner as early as 1905 to be due to lack of pancreatic ferments. It may be followed by volvulus and may lead, according to Lelong, Petit, Le Tan Vinh and Borniche (1950), to secondary atresia of the small bowel. In fibrocystic disease of the pancreas the degree of involvement of the pancreas and its ability to secrete pancreatic ferments must influence the manifestations of the disease; absence of secretion in the late intra-uterine period may lead to meconium ileus, partial lack to meconium retention, while deficient secretion in infancy may result in the coeliac syndrome or obstruction due to inspissation of bowel contents as in these cases.

It should be noted that vomiting unassociated with coughing, which is not an uncommon symptom of this disease, may be due to such an abnormality of small bowel contents. This is supported by the report of Rasor and Stevenson (1941) of a boy suffering from fibrocystic disease of the pancreas in whom vomiting was a persistent feature. At postmortem examination at 1 year of age, the intestinal contents were found to be of a putty-like consistency. As Levy (1951) suggests, the younger and more debilitated children are liable to this form of intestinal obstruction as older children with their more powerful intestinal peristalsis can probably propel the abnormal faeces through the ileocaecal valve.

In the case reported the intestinal obstruction followed closely the cessation of pancreatin therapy and was possibly relieved by the oral administration of pancreatin. This suggests a further use for pancreatin which has been advocated by Andersen (1945) as a form of replacement therapy. Newer preparations of enteric-coated pancreatin granules of three times the strength of the B.P. preparation are fairly well tolerated and effective in improving the absorption of food as well as the consistency and odour of the stools.

The occurrence of intestinal obstruction as a late 
complication of fibrocystic disease of the pancreas must be fully recognized. Laparotomy will confirm the obstruction is due to inspissation of small bowel contents and an ileostomy should be performed because the site of maximal obstruction is in the region of the ileocaecal valve and as this procedure has proved successful in cases of meconium ileus reported by Bodian (1952) and Hiatt and Wilson (1948). By this route, freshly prepared pancreatin can be irrigated into the small bowel while pancreatin is also given by gastric tube after aspiration. By these means, the abnormal bowel contents may be softened and liquefied thus relieving the intestinal obstruction.

\section{Summary}

A case of intestinal obstruction due to inspissated small bowel contents at 15 months of age is reported. Occurring as a late complication of fibrocystic disease of the pancreas the similarity to meconium ileus is discussed. Attention is drawn to the condition which is best treated by ileostomy and pancreatin therapy.

I am grateful to Dr. A. A. H. Gailey and Mr. Ian Fraser for permission to publish this case.

\section{REFERENCES}

Andersen, D. H. (1938). Am

Bodian (1945). Ibid., 69, 221 .

Bodian, M. (1952). Fibrocystic Disease of the Pancreas. London.
Hiatt, R. B. and Wilson, P. E. (1948). Surg. Gynec. Obstet., 87, 317. Hiatt, R. B. and Wilson, P. E. (1948). Surg. Gynec. Obstet.,
Landsteiner, R. (1905). Zbl. allg. Path. path. Anat., 16, 903.

Lelong, M., Petit, P., Le Tan Vinh and Borniche (1950). Arch. franc. Pédiat., 7, 225.

Levy, E (1951). Archives of Disease in Childhood, 26, 335 .

Rasor, R. and Stevenson, C. (1941). Rocky Mtn med. J., 38, 218. 